\title{
Increased expression of CYP1A1 and CYP1B1 in ovarian/peritoneal endometriotic lesions
}

\author{
Carla A Piccinato ${ }^{1,2}$, Rosa M Neme ${ }^{1,3}$, Natália Torres ${ }^{1}$, Lívia Renta Sanches ${ }^{4}$, \\ Priscilla Bento Mattos Cruz Derogis ${ }^{4}$, Heloísa F Brudniewski ${ }^{1,3}$, \\ Júlio C Rosa e Silva ${ }^{2}$ and Rui A Ferriani² \\ ${ }^{1}$ Hospital Israelita Albert Einstein, São Paulo, Brazil, ${ }^{2}$ Department of Gynaecology and Obstetrics, \\ School of Medicine of Ribeirão Preto, Universidade de São Paulo, São Paulo, Brazil, \\ ${ }^{3}$ Centro de Endometriose São Paulo, Av. República do Líbano, São Paulo, Brazil and ${ }^{4}$ Department of Clinical \\ Pathology, Hospital Israelita Albert Einstein, São Paulo, Brazil
}

Correspondence should be addressed to C A Piccinato; Email: cpiccinato@uwalumni.com

\begin{abstract}
Endometriosis is an estrogen-dependent disease affecting up to $10 \%$ of all premenopausal women. There is evidence that different endometriosis sites show distinct local estrogen concentration, which, in turn, might be due to a unique local estrogen metabolism. We aimed to investigate whether there was a site-specific regulation of selected enzymes responsible for the oxidative metabolism of estrogens in biopsy samples and endometrial and endometriotic stromal cells. Cytochrome P450 (CYP) 1A1 and CYP1B1 mRNA and protein expressions in deep-infiltrating (rectal, retossigmoidal, and uterossacral) lesions, superficial (ovarian and peritoneal) lesions, and eutopic and healthy (control) endometrium were evaluated by real-time PCR and western blot. Using a cross-sectional study design with 58 premenopausal women who were not under hormonal treatment, we were able to identify an overall increased CYP1A1 and CYP1B1 mRNA expression in superficial lesions compared with the healthy endometrium. CYP1A1 mRNA expression in superficial lesions was also greater than in the eutopic endometrium. Interestingly, we found a similar pattern of CYP1A1 and CYP1B1 expression in in vitro stromal cells isolated from ovarian lesions $(n=3)$ when compared with stromal cells isolated from either rectum lesions or eutopic endometrium. In contradiction, there was an increased half-life of estradiol (measured by HPLC-MS-MS) in ovarian endometriotic stromal cells compared with paired eutopic stromal endometrial cells. Our results indicate that there is a site-dependent regulation of CYP1A1 and CYP1B1 in ovarian/peritoneal lesions and ovarian endometriotic stromal cells, whereas a slower metabolism is taking place in these cells.

Reproduction (2016) 151 683-692
\end{abstract}

\section{Introduction}

Endometriosis is the most commonly diagnosed disease at reproductive age, affecting $5-10 \%$ of all premenopausal women (Eskenazi \& Warner 1997). This incidence increases to up to $50 \%$ in women with infertility, pelvic pain, or both (Eskenazi \& Warner 1997, Giudice \& Kao 2004). Endometriosis is defined as the presence of endometrial glands and stroma in extrauterine sites (Giudice \& Kao 2004). Although immune system and environmental endocrine disruptive compounds are considered to play a role in the development of endometriosis, estrogen dependency is considered the key characteristic of endometriosis, on which most effective clinical treatments are based (Ferrero et al. 2009, Giudice 2010).

Estrogen can stimulate endometriosis lesions by triggering estrogen receptor-mediated signal transduction or translation, resulting in increased
DNA synthesis and cell proliferation. The estrogen that is locally available to act at the endometrium and endometriotic lesions originates either from circulating estrogens or from its in situ production (Huhtinen et al. 2012). The fine balance between synthesis and metabolism, i.e., estrogen production and local inactivation, determines the net local estrogen availability. Such balance is directly associated with the expression and activity of steroid-synthesizing vs. metabolizing enzymes. Amongst the latter, the oxidative metabolizing enzymes, referred to as the cytochrome P450 family (CYPs), are likely candidates for modulating the action of estrogens, such as estradiol and estrone. The CYPs convert these steroids into hydroxyestrogens that have reduced estrogenic activity with variable extent of binding affinity to the estrogen receptors (Lee et al. 2001, Zhu \& Conney 1998). Although most CYPs were previously considered restricted to liver 
(Martucci \& Fishman 1993), it has long been known that CYP1A1 and CYP1B1 are widely expressed in extrahepatic tissues, such as the endometrium (Hakkola et al. 1997, Bofinger et al. 2001, Pavek \& Dvorak 2008, Singh et al. 2008, Hevir et al. 2013). CYP1A1 catalyzes $2-\mathrm{OH}$ hydroxylation in the endometrium and consequently plays an important role in producing less estrogenic metabolites, the 2-OH-estrogens (Tsuchiya et al. 2005). Conversely, CYP1B1 originates 4-OH catechol estrogen, a hydroxyestrogen, that acts as a strong agonist of ER receptors (i.e., active estrogens) and that is also potentially carcinogenic after being oxidized to quinones (Zhu \& Conney 1998). Therefore, the expression of CYP1A1 and CYP1B1 appears to be critical determinants of the metabolism and toxicity of estrogens, according to the prevalent metabolite that has been produced.

There are evidences that different lesion sites show distinct local estrogen concentration (Huhtinen et al. 2012), which, in turn, might be due to their unique local estrogen metabolism. Indeed, the regulation of expressed/active CYPs in association with other metabolizing enzymes determines the net estrogenic activity of the endometrium and may be implicated in estrogen-dependent endometrial diseases, such as endometriosis. The role of oxidative metabolism of estrogens in the pathogenesis of endometriosis is just started to be investigated. So far, it is known that CYP1A1 is highly expressed in ovarian lesions, whereas CYP1B1 has similar expression levels in the endometrium and ovarian lesions (Bulun et al. 2006, Singh et al. 2008, Hevir et al. 2011). We hypothesize that because there are distinct local estrogen concentrations (Huhtinen et al. 2012), different sites have distinct expression of CYP1 A1 and CYP1B1. Thus, the study of the expression of the CYPs in different lesion sites and in the endometrium could help to understand the pathogenesis and progression of endometriosis.

We propose to investigate whether a site-specific regulation of CYP1A1 and CYP1B1 mRNA and protein expression occur in deep-infiltrating (rectal, retossigmoidal, and uterossacral) endometriosis lesions compared with superficial (ovarian and peritoneal) lesions and to the eutopic and control endometrium. Gene expression of these CYPs and the activity of estrogen metabolizing enzymes were further evaluated in stromal cell cultures derived from endometrium or endometriosis lesions.

\section{Materials and methods}

The study was approved by the Committee on Human Research of the Hospital Albert Einstein (FR-30468, 12/16/2009; São Paulo, Brazil), which is affiliated to the Ethics Committee of the Brazilian Ministry of Health (CONEP). Informed consent was obtained from all persons enrolled in the study.

\section{Study design - biopsy specimens}

Specimens of eutopic endometrium and lesions (superficial and deep-infiltrating lesions) were collected from 42 patients $(32.2 \pm 6.7$ years old) undergoing laparoscopy. Control endometrial samples were obtained following tube ligation from an additional 16 patients $(33.9 \pm 0.7$ years old), and the absence of endometriosis was confirmed during surgery. The patients were classified according to the revised classification criteria of the American Society for Reproductive Medicine (ASRM 1996). The patients enrolled donated specimens to form our sample cohort that includes proliferative and luteal phase samples of healthy (control) endometrium $(n=25)$, eutopic endometrium $(n=24)$, and superficial $(n=18)$ and deep-infiltrating $(n=16)$ lesions of endometriosis patients. The classification of superficial lesions included ovarian and peritoneal lesions, whereas deep-infiltrating lesions stand for lesions collected from the rectosigmoid colon, urethra, uterosacral ligament, and rectum. The specimens were collected during surgery and immediately placed into RNA stabilization solution (RNA Later; Qiagen) or liquid nitrogen and kept at $-80^{\circ} \mathrm{C}$ until RNA/protein extraction. Diagnosis of endometriosis was confirmed histologically by experienced gynecological pathologists. All subjects had not taken hormone medication for at least 3 months before biopsy collection. The exclusion criteria were the presence of other reproductive disorders (such as polycystic ovary syndrome) or any tumors. The phase of the menstrual cycle was determined by the expected day of menstrual cycle provided by the women compared with the measurements of serum levels of estradiol and progesterone (follicular phase: $\mathrm{P} 4<1.0 \mathrm{ng} / \mathrm{mL}$, luteal phase: $\mathrm{P} 4>1.0 \mathrm{ng} / \mathrm{mL}$ ). The gene and protein expressions of CYP1A1 and CYP1B1 were compared among tissues from different sites and classified according to the cycle phase. There were paired and non-paired samples in this cohort because some of the patients donated one sample, and others donated two or more samples. Thus, a subset of endometriosis patients $(n=13)$ provided paired endometrium and lesions that were analyzed separately for CYP gene expression.

\section{RNA isolation, cDNA synthesis, and real-time PCR}

Total RNA was extracted using the RNA-Protein Kit (MachereyNagel, Duren, Germany) after disrupting the tissue using a Precellys Automated Homogenizer with a CK28 beads (Bertin Technologies, Montigny le Bretonneux, France). For cDNA synthesis, $200 \mathrm{ng}$ total RNA were reverse transcribed in a final $25 \mu \mathrm{L}$ optimized reaction volume using a SuperScript III Reverse Transcriptase kit (Invitrogen, San Diego, CA) with oligo(dT) primers. Primer sequences are described in Table 1. Real-time PCR analyses of the genes of interest (CYP1A1 and CYP1B1) and three housekeeping genes (ACTIN, $G A P D H$, and HPRT) were performed using gene-specific primers for amplification by SybR Green assay protocol (Life Technologies). Quantification was accomplished with a Life Technologies 7500 real-time RT-PCR machine using SybR Green PCR Master Mix (Life Technologies) and standard 
Table 1 Sequence of primers.

\begin{tabular}{lllc}
\hline Gene & \multicolumn{2}{l}{ Primer sequence } & Product length \\
\hline CYP1A1 & F: & 5'-TTCCCTGATCCTTGTGATCC-3' & $109 \mathrm{pb}$ \\
& R: & 5'-GGAGATTGGGAAAAGCATGA-3' & \\
CYP1B1 & F: & 5'-CGCAACCAAGTGTGCTAAAA-3' & $75 \mathrm{pb}$ \\
& R: & 5'-GAAACCCTGCTTCATTTCCA-3' & \\
k-Actin & F: & 5'-CCCAGCACAATGAAGATCAA-3' & $103 \mathrm{pb}$ \\
& $\mathrm{R}:$ & 5'-ACATCTGCTGGAAGGTGGAC-3' & \\
HPRT & F: & 5'-TGCTCGAGATGTGATGAAGG-3' & $95 \mathrm{pb}$ \\
& R: & 5'-AATCCAGCAGGTCAGCAAAG-3' & \\
GAPDH & F: & 5'-ATTGCCCTCAACGACCACTT-3' & $64 \mathrm{pb}$ \\
& R: & 5'-TGCTGTAGCCAAATTCGTTGTC-3' & \\
\hline
\end{tabular}

thermocycling parameters recommended by Life Technologies. The RT-PCR samples were run in duplicates using $1.2 \mu \mathrm{L}$ cDNA in a final reaction of $15 \mu \mathrm{L}$. A pool of endometrial tissue and HepG2 cells (obtained from the Cell Bank of Rio de Janeiro, Brazil) was used as reference sample (or calibrator sample) for the calculations of CYP expression in the biopsies and stromal cells. The gene expression normalization factor for each sample was generated by geNorm software and was calculated based on the geometric mean of all the three selected reference genes (Vandesompele et al. 2002). Results are expressed as the mean fold change of the normalized gene expression relative to the calibrator sample, using the $\Delta \Delta \mathrm{Ct}$ method (Livak \& Schmittgen 2001).

\section{Western blotting}

Total proteins were extracted after total RNA isolation using the RNA-Protein Kit (Macherey-Nagel, Düren, Germany). Protein concentration was determined by the BCA protein assay kit (Bio-Rad). Western blot analysis was performed using antiCYP1A1 antibody (1:5000, ab3568; Abcam) \& anti-CYP1B1 Sigma (1:2000, HPA026863, Prestige Antibodies; SigmaAldrich). Samples $(\sim 50 \mu \mathrm{g})$ were next separated on a $10 \%$ acrylamide gel, followed by their transfer to nitrocellulose Hybond membranes (GE Healthcare). After blocking with $5 \%$ skimmed milk $(1 \mathrm{~h})$, membranes were incubated with the primary antibodies overnight at $4^{\circ} \mathrm{C}$, then with the secondary antibodies (1:5000, horseradish peroxidase-conjugated antirabbit IgG [sc2030] or anti-mouse IgG [sc2031] antibodies; Santa Cruz Biotechnology) for $1 \mathrm{~h}$ at room temperature. Immunoreactive bands were detected using the ECL system (GE Healthcare). Anti- $\beta$-actin antibodies (A1978; SigmaAldrich) were used to account for cellular protein loading. Band intensity was determined by densitometric analysis using TotalLab Quant software Version 2008 (TotalLab Ltd, Newcastle upon Tyne, UK).

\section{Serum hormone assays}

Serum levels of estradiol and progesterone were determined by chemiluminescence assays, using the Diagnostic Products Corporation Immulite 2000 and the respective kits for Immulite 2000 (Siemens Healthcare). The assay sensitivity reached $0.1 \mathrm{ng} / \mathrm{mL}$ for progesterone and $15 \mathrm{pg} / \mathrm{mL}$ for estradiol. The intra- and inter-assay coefficients of variation were approximately 6.6 and $9.7 \%$, and 9.6 and $12.2 \%$, respectively, for the estradiol and progesterone assay. These assays were carried out at the Gynaecology and Obstetrics Laboratory of the Ribeirão Preto University Hospital and were performed by the same technician.

\section{Study design - in vitro}

The same criteria used for the biopsy specimen study were used to select endometriosis subjects for stromal cell culture experiments. Stromal cells were isolated from eutopic and ectopic (rectal and ovarian endometriotic lesion) endometrium of nine endometriosis patients. Stromal cell compartment expresses CYPs (Singh et al. 2008) and, so far, have been neglected as a pivotal compartment that have a role in estrogen homeostasis. Therefore, we aimed to understand how the mRNA expression of CYP1A1 and CYP1B1 in these cells is and whether the altered expression of the CYPs would produce altered estradiol metabolism. Primary culture of the stromal cells from endometrium and endometriotic lesions (ovarian and rectal) was performed based on Klemmt et al.'s study with few modifications (Klemmt et al. 2007). Briefly, tissue digestion was performed with collagenase type I (02674; Sigma-Aldrich, Saint Louis, USA) for $40 \mathrm{~min}$ at $37^{\circ} \mathrm{C}$. Cells were isolated in Dulbecco's Minimum Essential Medium (DMEM; Gibco Laboratories, Grand Island, NY, USA) and were passed through a set of sieves (250 and $35 \mu \mathrm{m}$ mesh sieves). Culture and expansion were performed in DMEM supplemented with $10 \%$ FBS and $1 \%$ antibiotic/antimycotic solution (Gibco Laboratories, Grand Island, NY, USA). Stromal cells at early passage (fourth - fifth passage) were seeded in duplicate at low density (approximately $600 \mathrm{cells} / \mathrm{cm}^{2}$ ) in DMEM with $10 \% \mathrm{FBS}$ and $1 \%$ antibiotic/antimycotic solution (Gibco Laboratories, Grand Island, NY, USA). After overnight attachment, cells were incubated for 3 days in a phenol red-free medium (M199; Gibco Laboratories, NY, USA) supplemented with $2 \%$ charcoal-stripped serum (Invitrogen) at $37^{\circ} \mathrm{C}$ in an incubator with $5 \% \mathrm{CO}_{2}$. These cells were harvested for RNA isolation (for the CYP expression study) or were used for estradiol decay experiments (for the estradiol metabolism study). RNA isolation, cDNA synthesis, and PCR amplification of stromal cells were performed as described above for biopsy specimens.

\section{Estradiol decay analysis}

Fresh phenol red-free M199 medium supplemented only with $10 \mathrm{nM} 17 \beta$-estradiol $(0.01 \% \mathrm{v} / \mathrm{v}$; Sigma-Aldrich) and without charcoal-stripped serum was added to the stromal cell cultures. A time-course analysis of estradiol decay was undertaken by collecting $500 \mu \mathrm{L}$ medium from duplicate wells at 0,1 , and $2 \mathrm{~h}$. The experiments were run in pairs of stromal cell cultures from the same patient but of different sources: eutopic and ectopic (ovarian lesions) endometrium ( $n=3$ in duplicate wells; paired stromal cell samples). At the end of the experiment, the cells were counted by the trypan blue exclusion method to adjust for estradiol decay according to the final number of cells. 

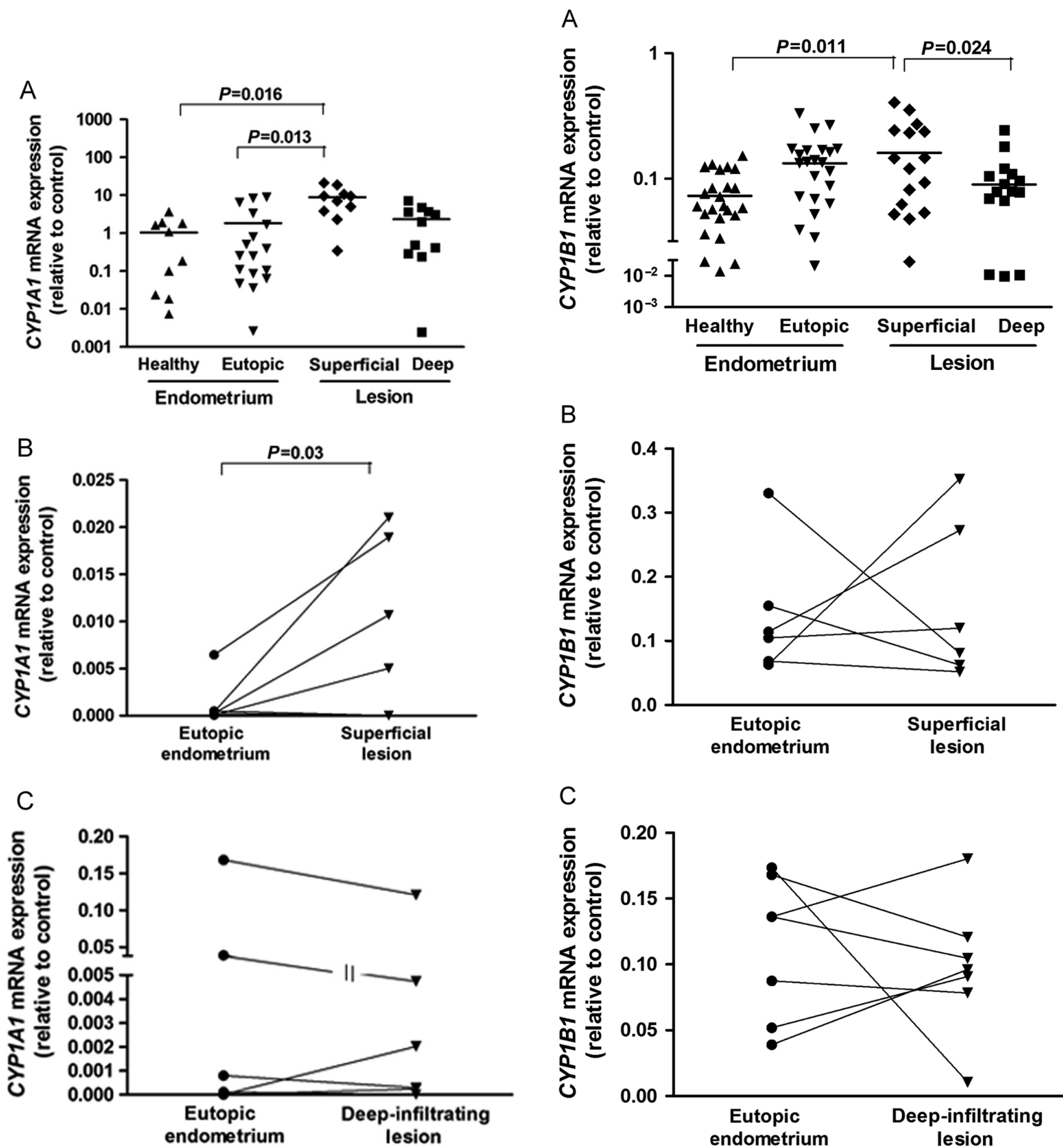

Figure 1 CYP1A1 mRNA levels are increased in superficial lesions. The gene expression levels of CYP1A1 (A, B and C) were analyzed by real-time PCR in healthy and eutopic endometrium and superficial and deep-infiltrating lesions. Relative gene expression levels were normalized by geNorm algorithm and scatter plots represent the mean fold change of the normalized gene expression relative to a calibrator sample. Paired analyses were also performed in 13 patients who provided eutopic endometrium and superficial (B) or deepinfiltrating lesions (C). Statistical differences between groups are indicated by $P$ values.

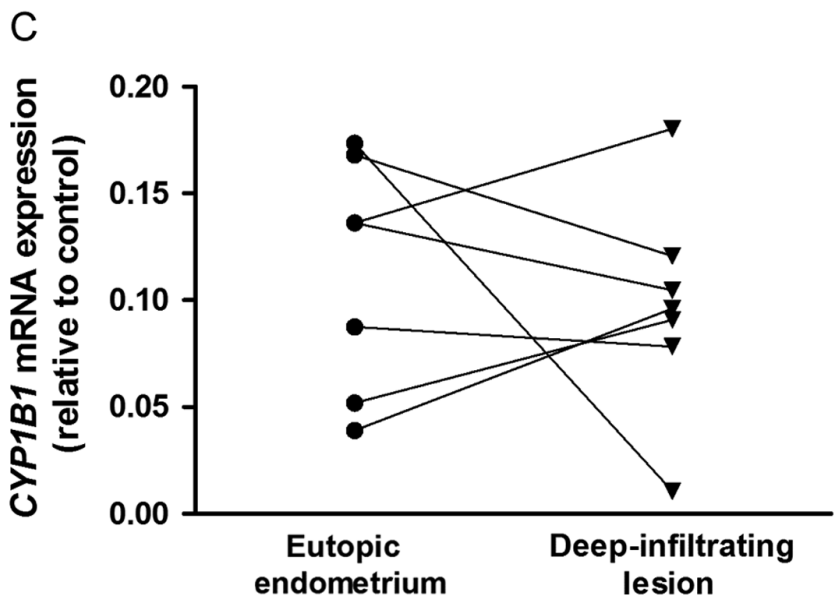

Figure 2 CYP1B1 mRNA levels are increased in superficial lesions. The gene expression levels of CYP1B1 (A, B and C) were analyzed by real-time PCR in healthy and eutopic endometrium and superficial and deep-infiltrating lesions. Relative gene expression levels were normalized by geNorm algorithm and scatter plots represent the mean fold change of the normalized gene expression relative to a calibrator sample. Paired analyses were also performed in 13 patients who provided eutopic endometrium and superficial (B) or deepinfiltrating lesions $(C)$. Statistical differences between groups are indicated by $P$ values. 
Inhibition of CYP1B1 and CYP1A1 activity was performed by using flutamide (F-9397; Sigma-Aldrich) at $100 \mathrm{nM}$ as a specific inhibitor of CYP1 family (Rochat et al. 2001), $10 \mathrm{~min}$ before adding $17 \beta$-estradiol to the medium.

\section{Measurements of estradiol in cell culture medium by liquid chromatography-tandem mass spectrometry (LC-MS/MS)}

The $17 \beta$-estradiol levels in the medium were determined using the LC-MS/MS method, as described for estradiol determination previously (Piccinato et al. 2016). After protein precipitation, medium samples containing a deuterated internal standard (17-hydroxyprogesterone-d8, CDN Isotopes Inc., Pointe Claire, QC, Canada) were filtered and injected onto a Kinetex C18 HPLC column (Phenomenex, Torrance, CA, USA), using an Agilent 1260 LC system (Agilent Technologies). The LC system was coupled to an AB SCIEX QTRAP 5500 tandem mass spectrometer (AB SCIEX, Concord, ON, Canada) fitted with an atmospheric pressure chemical ionization source. The linearity of the method was assessed by performing three replicate analyses with eight different concentrations ranging from 0.09 to $12 \mathrm{ng} / \mathrm{mL}\left(R^{2}=0.997\right)$. The inter-assay coefficients of variation were lower than $15 \%$ when analyzing the quality controls, with accuracy ranging from 96 to $105 \%$. 17 $\beta$-Estradiol levels were analyzed by the Analyst software version 1.6 (AB SCIEX, Concord, ON, Canada).

\section{Immunofluorescence of endometriotic stromal cells}

Cultured stromal cells at fourth to fifth passages were fixed with $4 \%$ paraformaldehyde in PBS followed by cellular permeabilization with and $0.1 \%$ Triton-X100 and incubation at $4{ }^{\circ} \mathrm{C}$ into a blocking solution, consisting of PBS with $3 \%$ bovine serum albumin. Subsequently, cells were incubated overnight with primary antibodies for vimentin (1:200, M0725; Dako) and cytokeratin 18 (1:300, CK18; Dako) in separate slides. Mouse IgG antibodies instead of primary antibodies were used as negative controls. Fluorescein isothiocyanate (FITC)-conjugated secondary antibody (1:400, M4413; Cell Signaling) was used for fluorescence staining. Nuclear staining was performed by mounting slides with coverslips using Vectashield medium containing DAPI (Vector Laboratories, Burlingame, CA, USA). Cell images were acquired with an IX-51 inverted microscope (Olympus).

\section{Calculations and statistical analysis}

All gene and protein expression data were analyzed using SAS software version 6.11 (Statistical Analysis System Institute Inc., Cary, NC, USA) and the Generalized Additive Models for Location Scale and Shape (GAMLSS) in R software (Stasinopoulos \& Rigby 2007) respectively. A random effect of patients was also used in all the analysis to account for paired and non-paired samples. Gene expression data were log-transformed to reduce skewness. Regression analysis with fixed effects of experimental groups and cycle and interactions between them were performed using MIXED procedures of SAS. A post hoc Tukey's test was applied when the main effect was described as significant. In the culture experiments, paired $t$-test analysis was performed in the gene expression data after calculating fold change in relation to control (vehicle-treated cell culture). Protein expression of CYP1A1 and CYP1B1 was determined by the generalized mixed model accounting for zero-inflation with Gamma distribution and a log link in $\mathrm{R}$ (GAMLSS package).
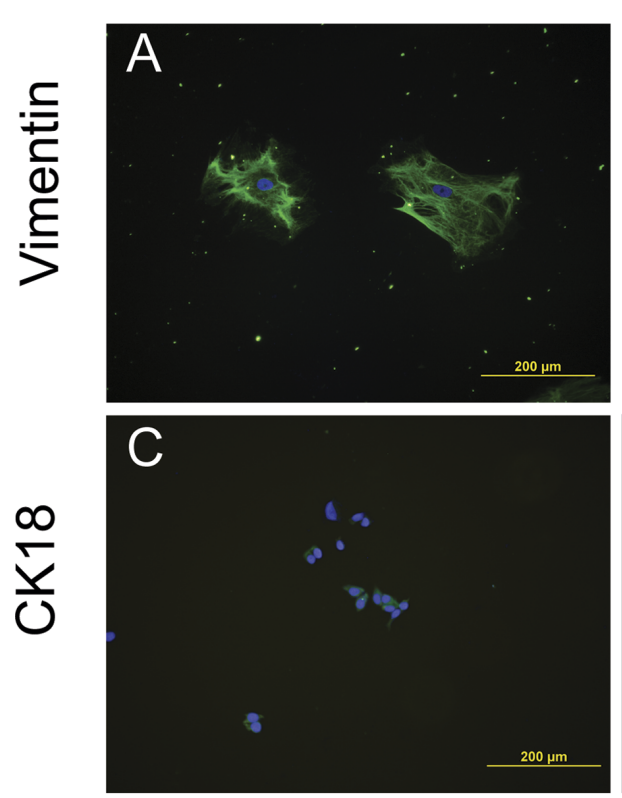

Positive control
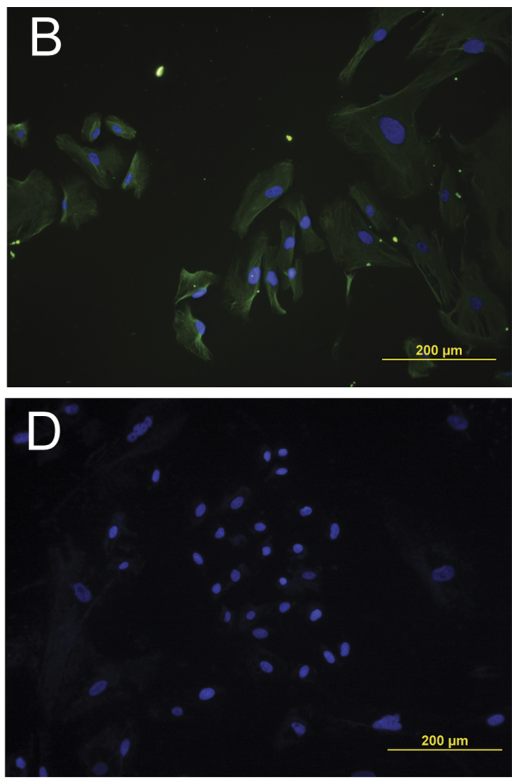

Stromal cells
Figure 3 Morphological features of endometriotic stromal cells. Positive control of vimentin (A, bone marrow mesenchymal stem cells) and representative photomicrographs of ovarian endometriotic stromal cells at the fifth passage in culture showing typical elongated and spindle-like morphology and positive staining for vimentin by immunofluorescence (B). Control of positive CK18 staining (C, MCF-7 cells) and the representative endometriotic stromal cells that show CK18-negative staining (D). The secondary antibody used in both assays was FITC-labeled and the nuclei were stained with DAPI. Scale bar: $200 \mu \mathrm{M}$. 
The fractional rate constant of estradiol clearance at time points $0,5,30,60$, and 120 min following addition of $10 \mathrm{nM}$ $17 \beta$-estradiol to the culture medium was determined using the equation: $\mathrm{N}(t)=\mathrm{N}^{-k \mathrm{e}} \mathrm{e}^{-}$, where $\mathrm{N}$ is the estradiol at time $t, \mathrm{~N} 0$ is the initial value, $k$ is the first-order fractional rate constant, $t$ is time, and $e$ is the base of natural logarithms. Half-lives were calculated using the natural logarithms of 2 divided by $k$. In all analyses, $P<0.05$ was considered to be statistically significant.

\section{Results}

\section{Differential expression of CYP1A1 and CYP1B1 among sources of endometrial tissue}

Although samples were classified according to the two main phases of the menstrual cycle, no differences in CYP1A1 expression could be detected between follicular and luteal phases. Nevertheless, as shown in Fig. 1A, significant differences in CYP1A1 mRNA expression were observed between superficial lesions vs eutopic endometrium $(P=0.013)$ and between superficial lesions vs. healthy endometrium $(P=0.016)$. However, no differences in CYP1A1 mRNA expression were seen between distinct sources of lesions, as deepinfiltrating lesions presented similar levels of expression as superficial ovarian lesions. Similarly, when only samples from the same patients were analyzed, superficial lesions showed consistent greater CYP1A1 expression, compared with the eutopic endometrium (Fig. 1B; $P=0.03$ ) and overall deep-infiltrating lesions and the eutopic endometrium showed similar CYP1A1 expression (Fig. 1C). Although superficial lesions showed increased levels of CYP1A1 gene expression in comparison to both eutopic and healthy endometrium, protein expression of CYP1A1 was similar in the tissue samples obtained from the different sites and at both cycle phases, suggesting posttranscriptional regulation of CYP1A1 synthesis (Supplementary Fig. 1, see section on supplementary data given at the end of this article).

Similarly to CYP1A1, no effect of cycle phase was apparent in our samples with regard to CYP1B1 gene and protein expression. As shown in Fig. 2A, CYP1B1 mRNA levels were higher in the superficial lesions compared with deep-infiltrating lesions $(P=0.024)$ and also compared with healthy endometrium samples $(P=0.011)$. However, in contrast to CYP1A1, no variation in CYP1B1 gene expression was detected when paired eutopic endometrium and superficial lesions (Fig. 2B) or deep-infiltrating lesions were analyzed (Fig. 2C). In relation to CYP1B1 protein expression, no difference among the sampled tissues was seen using the western blot method (Supplementary Fig. 1).

\section{Constitutive expression of CYP1A1 and CYP1B1 in endometrial and endometriotic stromal cells in culture}

Endometrial and endometriotic stromal cells around the fifth passage were characterized as such Reproduction (2016) 151 683-692 by immunofluorescence staining with vimentin (a cytoskeleton stromal cell marker) and by their negative immunoreactivity for the epithelial cell marker cytokeratin 18, indicating the purity of the isolated stromal cells (Fig. 3). Constitutive expression of CYP1A1 was observed in all cell groups, although ovarian endometriotic stromal cells showed a sharp increased CYP1A1 expression, compared with stromal cells from eutopic endometrium and rectal lesions (Fig. 4A, $P=0.003$ and $P=0.015$ respectively). A pronounced
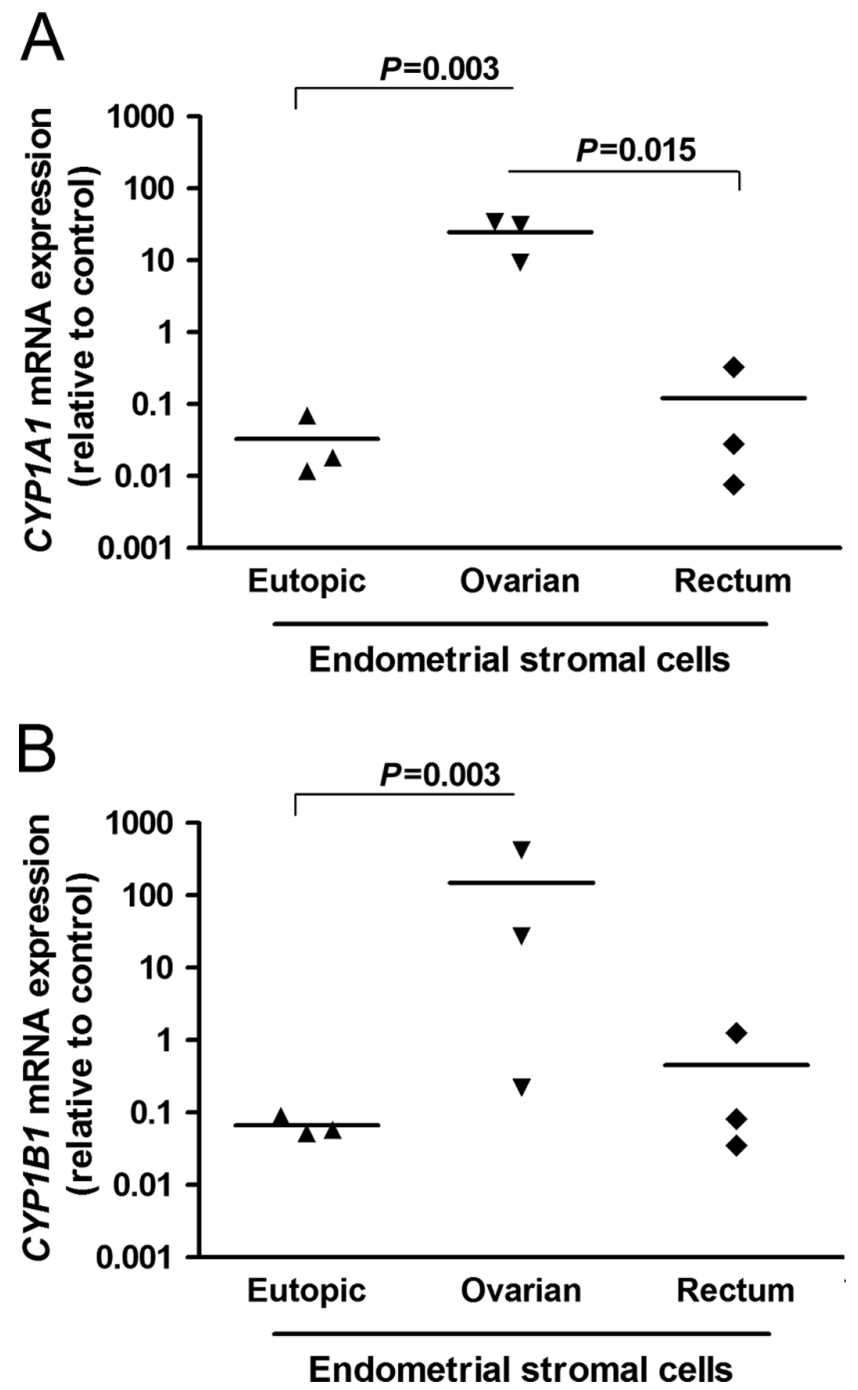

Figure $4 \quad C Y P 1 A 1$ and $C Y P 1 B 1 \mathrm{mRNA}$ levels are increased in ovarian endometriotic stromal cells. The baseline mRNA levels of CYP1A1 (A) and CYP1B1 (B) were evaluated by real-time PCR, in stromal cells from eutopic and ectopic (ovarian and rectal lesions) endometria cultivated in steroid-free conditions $(n=3$ per group, in duplicate). The average of each duplicate cell culture that is represented in the scatter plot was calculated relative to the geNorm algorithm and to the calibrator sample, using the $\Delta \Delta \mathrm{Ct}$ method and shown as LSM \pm S.E.M. Significant differences between groups (eutopic versus ovarian endometriosis versus deep-infiltrating stromal cells) are indicated in the figures by the $P$ values. 
increased CYP1B1 expression was also detected in ovarian endometriotic stromal cells (Fig. 4B; $P=0.003$ ) in relation to stromal cells from the eutopic endometrium.

\section{Estradiol decay in eutopic and ectopic (ovarian) endometrial stromal cells}

Because we found increased expression of selected CYPs in the ovarian endometriosis stromal cells, we further investigated whether these cells also have altered estradiol metabolism. As presented in Table 2, the fractional rate constant for estradiol was lowest in ovarian endometriotic stromal cells compared with paired stromal cells from eutopic endometrium $(P=0.008)$. Furthermore, the half-life of estradiol in stromal cells of ovarian endometriotic lesions was almost double the one in stromal cells from the eutopic endometrium $(P=0.025)$. These differences were consistent in all paired cell lines. The selected CYP1 competitive inhibitor, flutamide, was used to block CYP1-mediated estradiol metabolism in order to test whether it would be possible to modulate estradiol halflife by reducing CYP1 activity. Treatment with $100 \mathrm{nM}$ flutamide did not affect estradiol half-life in either cell line, although a group effect was also observed (Fig. 5; eutopic endometrial vs. ectopic endometriotic ovarian stromal cells, $P=0.03$ ).

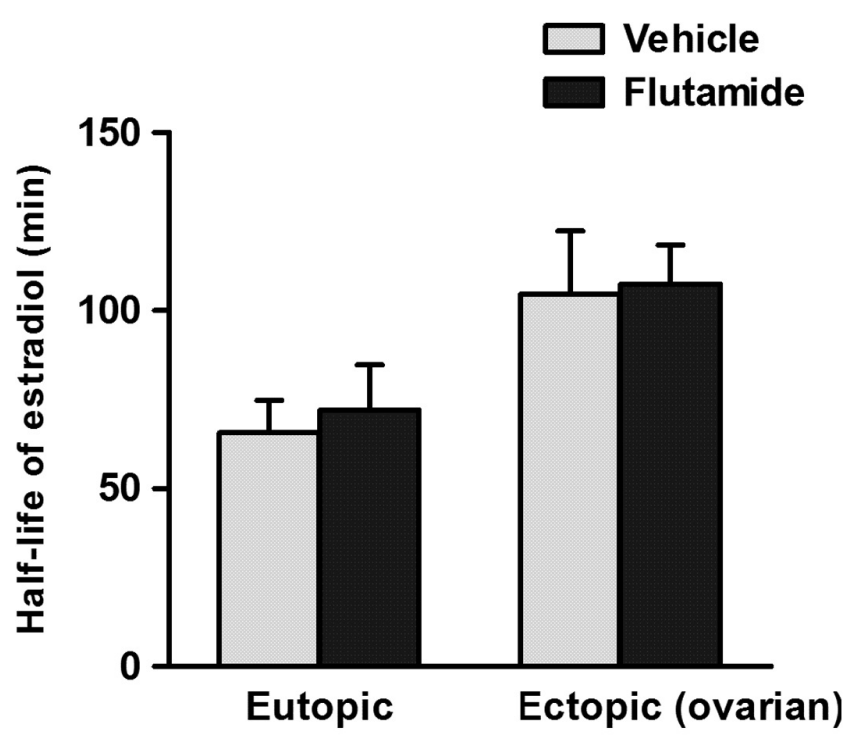

Endometrial stromal cells

Figure 5 Lack of inhibition of estradiol decay in endometriotic stromal cells. Lack of effect of $100 \mathrm{nM}$ flutamide in eutopic and ectopic (ovarian lesions) endometrial stromal cells by LC-MS/MS, and an effect of time was observed ( $P$ values between groups at each time point are indicated in the figure; $n=3$, in duplicate). After the end of the culture period ( $2 \mathrm{~h}$ ), there was a more pronounced decay of estradiol in the stromal cells of eutopic lesions than in cells from ectopic ovarian endometrium, regardless of flutamide treatment $(P=0.03, n=3$ in duplicate).
Table 2 Estradiol half-life ( $\mathrm{min}$ ) and fractional rate constant of decay $(K)$ for estradiol in steroid-free cultures of endometrial stromal cells. The cells were isolated from the eutopic and ectopic (ovarian lesions) endometrium $(n=3)$. Independent cultures of stromal cells originating from three different individuals (paired eutopic and ectopic) were analyzed. Means and S.E.M. (in parenthesis).

\begin{tabular}{lccc}
\hline & \multicolumn{2}{c}{ Endometrial stromal cells } & \\
\cline { 2 - 3 } & Eutopic & Ectopic (ovarian) & $\boldsymbol{P}$ value \\
\hline $\begin{array}{l}\text { Fractional rate constant of } \\
\text { decay }(K)\end{array}$ & $0.0107(0.003)$ & $0.0065(0.002)$ & 0.008 \\
Half-life $(\mathrm{min})$ & $68.8(0.001)$ & $120(0.0008)$ & 0.025 \\
\hline
\end{tabular}

\section{Discussion}

This study is the first to investigate the expression pattern of CYP1A1 and CYP1B1 in distinct endometriosis lesion sites of the same population cohort. Our results indicate that there is site-dependent regulation of CYP1A1 and CYP1B1 in endometriosis, as shown by the augmented expression of both enzymes in superficial lesions in comparison to eutopic and deep-infiltrating lesions. Constitutive expression of CYP1A1 and CYP1B1 in the endometrium has been described by others (Bofinger et al. 2001, Murray et al. 2001, Hevir et al. 2011) and was also observed in this study, although no differences in the expression of CYP1A1 or CYP1B1 were noted between the endometrium samples from healthy patients and those taken from endometrium from patients with endometriosis. The major difference detected by our results highlights a greater expression of CYP1A1 and $C Y P 1 B 1$ in samples taken from superficial endometriotic lesions. In addition, the greater CYP1A1 and CYP1B1 expression showed in the in vitro ovarian endometriotic stromal cells reinforces the results observed in the biopsy specimens. These results are in part in agreement with previous studies where CYP1A1, but not CYP1B1, was increased in ovarian endometriosis samples (Singh et al. 2008, Hevir et al. 2013). In our sampling strategy, the samples classified as superficial included both ovarian and peritoneal lesions. This latter information and the greater sample size of this study may explain the discrepancies between this and previous works in regard to CYP1B1 expression. Still, our results on the enhanced expression of CYP1B1 in the ectopic (ovarian) endometrium are similar to those obtained in a previous study on a smaller number of patients (Singh et al. 2008). The consistent greater mRNA expression of CYP1A1 and CYP1B1 in superficial endometriosis lesions was not accompanied by seemingly comparative increase in protein expression in tissue extracts analyzed by the western blot technique, a finding that may suggest post-transcriptional regulation of CYPs in endometrial and endometriotic tissues, promoted by hormones, interleukins, microRNAs, or even heavy metals. Although discrepancies between mRNA and protein expression have been described before in CYPs, technical limitations in the methodologies used cannot be precluded. Cross-reactive antibodies binding to 
different CYP subfamilies may reduce their specificity and, overall, real-time PCR is considered a technique of higher sensitivity than western blotting. Yet, most studies show high correlation between activity of CYP and mRNA expression (Ciolino \& Yeh 1999), which justify the use of the latter as a parameter of the expression of these enzymes.

Additionally, we observed that CYP1B1 expression is much reduced in deep-infiltrating lesions compared with superficial endometriosis sites. This has not been described before, as no other previous study used a sampling cohort with all the variety of groups presented herein.

Even though we classified the endometrium samples according to the menstrual cycle, leading to the formation of two distinct groups of samples per site, no significant difference between cycle phases in the expression of CYP1A1 and CYP1B1 was observed in endometrial or endometriotic samples, from either healthy or endometriosis patients. This means that the expression of these selected CYPs do not fluctuate during the menstrual cycle in endometrial tissue under normal conditions. Similar results regarding CYP1B1 and CYP1A1 expression have been previously described in the healthy endometrium and in the endometrium of women with endometriosis (Hevir et al. 2011, 2013).

Differences in the regulation of CYPs at different sites of endometriosis can be influenced by the surrounding anatomical environment. The superficial lesions in which we found greater expression of CYP1A1 and CYP1B1 are surrounded by peritoneal fluid and peritoneal adipose tissue that contain a wide variety of compounds that may potentially regulate CYP expression (revised by Zanger \& Schwab 2013). Indeed, growing evidence suggests that the peritoneal fluid plays a critical role in the implantation and progression of endometriosis (revised by Koninckx et al. 1998). Peritoneal fluid contains detectable levels of dioxin or dioxin-like compounds such as 2,3,7,8-tetrachlorodibenzo-pdioxin (TCDD) depending on individual exposure (Cai et al. 2011) and it has been recently shown that the adipose tissue from the omentum in women with endometriosis contains a greater amount of dioxin-like compounds when compared with age and body mass index-matched controls (Martinez-Zamora et al. 2015). One can speculate that such compounds are involved in endometriosis as they can transcriptionally induce CYP1B1 and CYP1A1 in explant cultures of human endometrial or stromal cells (Bofinger et al. 2001), mostly by their interaction with nuclear receptors, as the resulting complexes bind to the regulatory region of the gene and modulate CYP expression (Waxman 1988). Selected nuclear receptors also preferentially modulate the expression of one CYP type and not of others. For instance, aryl hydrocarbon receptors (AHR) and estrogen receptors $\alpha$ (ESR1) and $\beta$ (ESR2) are more closely related to the CYP1A1 and CYP1B1 induction
(Zanger \& Schwab 2013). More recently, the presence of AHR has been detected in endometriosis (Huhtinen et al. 2014) and, remarkably, a greater expression of ESR1 was detected in ovarian lesions (Huhtinen et al. 2012). Hence, the expression of specific nuclear receptors associated with varied concentrations of steroid hormones or compounds at different sites may explain such described isoform-specific regulation of CYPs in the endometrium and endometriosis lesions. However, these aspects were not investigated in the present work, and research in this direction is certainly warranted.

Considering the data on the expression of CYP1A1 and CYP1B1 in the isolated stromal cell lines, augmented expression of both CYPs was seen in cells collected from ovarian endometriosis. Although it is not possible to directly relate the data collected in the stromal cell model with the whole tissue (biopsy specimen data), it is worth to emphasize that similar pattern was observed in both models. This may signify that even after several in vitro passages, the stromal cells are able to retain the expression pattern of the whole tissue, suggesting that epigenetic modifications might have occurred before isolating the cells, extending this phenotype through late in vitro passages. Interestingly, this 'retained memory' has been observed in other studies of isolated stromal endometrial cells (Tsai et al. 2001, Aghajanova et al. 2010). Paradoxically to the expression data, when we performed functional studies of estradiol metabolism with the same cells, a pronounced increase in estradiol half-life was observed in ovarian endometriotic stromal cells. These are novel data that have not been described before, particularly because so far steroidogenesis and steroid metabolism have been mostly attributed to the epithelial compartment. Yet, data from previous study in our laboratory (Piccinato et al. 2016) and the present work highlight the need of future studies regarding the impact of stromal cells on estradiol concentration in the endometrium and endometriotic lesions, as the role of different cellular compartments in the metabolism of estradiol has not been exploited.

Unexpectedly, flutamide did not alter CYP1mediated estradiol metabolism despite been added before estradiol and at ten-fold molar excess (in relation to estradiol) to stromal cells with known greater CYP1A1 and CYP1B1 expression. Although an extended dose-response experiment still need to be performed in order to optimize flutamide blockage in the stromal cells, it is possible that the lack of flutamide effect could be due the involvement of other estradiol metabolizing enzymes that may compensate the reduced activity of CYP1 by redirecting the substrate to other estradiol metabolizing enzymes.

In conclusion, selected CYPs were constitutively expressed in the endometriotic tissues and are also present in endometrial stromal cells; however, we observed a site-dependent regulation of CYP1A1 and CYP1B1. The increased expression of CYP1A1 and 
CYP1B1 in superficial samples could possibly be a response to a greater local estradiol concentration, commonly associated with endometriosis. However, as previously proposed (Hevir et al. 2013), altered expression and activity of oxidizing CYP enzymes might result, in turn, in excessive $\mathrm{OH}$-estrogens and reactive oxygen species formation and consequently stimulate endometriotic cell proliferation that occurs with the progression of the disease.

\section{Supplementary data}

This is linked to the online version of the paper at http://dx.doi. org/10.1530/REP-15-0581.

\section{Declaration of interest}

The authors declare that there is no conflict of interest that could be prejudiced to the impartiality of the research reported.

\section{Funding}

This work was supported by the São Paulo Research Foundation (FAPESP: 2010/02412-6) and CNPq/INCT-Hormones.

\section{Acknowledgments}

The authors would like to acknowledge Albina Verceze for the hormonal measurements and Vanessa B Pazzini, Gabriela dos S Hidalgo, Marta Diniz, Renata Silvério, and Isis Mozetic for their technical assistance during sample collection, storage, cell culture, and artwork services. The authors also thank Elivane da Silva Victor and Deisy Morselli Victor for statistical consulting and Ises A Abrahamsohn for kindly revising the manuscript.

\section{References}

Aghajanova L, Velarde MC \& Giudice LC 2010 Altered gene expression profiling in endometrium: evidence for progesterone resistance. Seminars in Reproductive Medicine 28 51-58. (doi:10.1055/s-0029-1242994)

Bofinger DP, Feng L, Chi LH, Love J, Stephen FD, Sutter TR, Osteen KG, Costich TG, Batt RE, Koury ST et al. 2001 Effect of TCDD exposure on CYP1A1 and CYP1B1 expression in explant cultures of human endometrium. Toxicological Sciences 62 299-314. (doi:10.1093/ toxsci/62.2.299)

Bulun SE, Cheng YH, Yin P, Imir G, Utsunomiya H, Attar E, Innes J \& Julie Kim J 2006 Progesterone resistance in endometriosis: link to failure to metabolize estradiol. Molecular and Cellular Endocrinology 248 94-103. (doi:10.1016/j.mce.2005.11.041)

Cai LY, Izumi S, Suzuki T, Goya K, Nakamura E, Sugiyama T \& Kobayashi H 2011 Dioxins in ascites and serum of women with endometriosis: a pilot study. Human Reproduction 26 117-126. (doi:10.1093/humrep/deq312)

Ciolino HP \& Yeh GC 1999 Inhibition of aryl hydrocarbon-induced cytochrome P-450 1A1 enzyme activity and CYP1A1 expression by resveratrol. Molecular Pharmacology 56 760-767.

Eskenazi B \& Warner ML 1997 Epidemiology of endometriosis. Obstetrics and Gynecology Clinics of North America 24 235-258. (doi:10.1016/ S0889-8545(05)70302-8)

Ferrero S, Venturini PL, Ragni N, Camerini G \& Remorgida V 2009 Pharmacological treatment of endometriosis: experience with aromatase inhibitors. Drugs 69 943-952. (doi:10.2165/00003495200969080-00001)

Giudice LC 2010 Clinical practice. Endometriosis. New England Journal of Medicine 362 2389-2398. (doi:10.1056/NEJMcp1000274)

Giudice LC \& Kao LC 2004 Endometriosis. Lancet 364 1789-1799. (doi:10.1016/S0140-6736(04)17403-5)

Hakkola J, Pasanen M, Pelkonen O, Hukkanen J, Evisalmi S, Anttila S, Rane A, Mantyla M, Purkunen R, Saarikoski S et al. 1997 Expression of CYP1B1 in human adult and fetal tissues and differential inducibility of CYP1B1 and CYP1A1 by Ah receptor ligands in human placenta and cultured cells. Carcinogenesis 18391-397. (doi:10.1093/carcin/18.2.391)

Hevir N, Sinkovec J \& Rizner TL 2011 Disturbed expression of phase I and phase II estrogen-metabolizing enzymes in endometrial cancer: lower levels of CYP1B1 and increased expression of S-COMT. Molecular and Cellular Endocrinology 331 158-167. (doi:10.1016/j.mce.2010.09.011)

Hevir N, Ribic-Pucelj M \& Rizner TL 2013 Disturbed balance between phase I and II metabolizing enzymes in ovarian endometriosis: a source of excessive hydroxy-estrogens and ROS? Molecular and Cellular Endocrinology 367 74-84. (doi:10.1016/j.mce.2012.12.019)

Huhtinen K, Desai R, Stahle M, Salminen A, Handelsman DJ, Perheentupa A \& Poutanen M 2012 Endometrial and endometriotic concentrations of estrone and estradiol are determined by local metabolism rather than circulating levels. Journal of Clinical Endocrinology \& Metabolism 97 4228-4235. (doi:10.1210/jc.2012-1154)

Huhtinen K, Saloniemi-Heinonen T, Keski-Rahkonen P, Desai R, Laajala D, Stahle M, Hakkinen MR, Awosanya M, Suvitie P, Kujari H et al. 2014 Intra-tissue steroid profiling indicates differential progesterone and testosterone metabolism in the endometrium and endometriosis lesions. Journal of Clinical Endocrinology \& Metabolism 99 E2188-2197. (doi:10.1210/jc.2014-1913)

Klemmt PAB, Carver JG, Koninckx P, McVeigh EJ \& Mardon HJ 2007 Endometrial cells from women with endometriosis have increased adhesion and proliferative capacity in response to extracellular matrix components: towards a mechanistic model for endometriosis progression. Human Reproduction 22 3139-3147. (doi:10.1093/ humrep/dem262)

Koninckx PR, Kennedy SH \& Barlow DH 1998 Endometriotic disease: the role of peritoneal fluid. Human Reproduction Update 4 741-751. (doi:10.1093/humupd/4.5.741)

Lee AJ, Kosh JW, Conney AH \& Zhu BT 2001 Characterization of the NADPH-dependent metabolism of 17 beta-estradiol to multiple metabolites by human liver microsomes and selectively expressed human cytochrome P450 3A4 and 3A5. Journal of Pharmacology and Experimental Therapeutics 298 420-432.

Livak KJ \& Schmittgen TD 2001 Analysis of relative gene expression data using real-time quantitative PCR and the 2(-Delta Delta C(T)) Method. Methods 25 402-408. (doi:10.1006/meth.2001.1262)

Martinez-Zamora MA, Mattioli L, Parera J, Abad E, Coloma JL, van Babel B, Galceran MT, Balasch J \& Carmona F 2015 Increased levels of dioxin-like substances in adipose tissue in patients with deep infiltrating endometriosis. Human Reproduction 30 1059-1068. (doi:10.1093/ humrep/dev026)

Martucci CP \& Fishman J 1993 P450 enzymes of estrogen metabolism. Pharmacology \& Therapeutics 57 237-257.

Murray GI, Melvin WT, Greenlee WF \& Burke MD 2001 Regulation, function, and tissue-specific expression of cytochrome P450 CYP1B1. Annual Review of Pharmacology and Toxicology 41 297-316.

Pavek P \& Dvorak Z 2008 Xenobiotic-induced transcriptional regulation of xenobiotic metabolizing enzymes of the cytochrome P450 superfamily in human extrahepatic tissues. Current Drug Metabolism 9 129-143. (doi:10.2174/138920008783571774)

Piccinato CA, Neme RM, Torres N, Sanches LR, Derogis PB, Brudniewski HF, Rosa ESJC \& Ferriani RA 2016 Effects of steroid hormone on estrogen sulfotransferase and on steroid sulfatase expression in endometriosis tissue and stromal cells. Journal of Steroid Biochemistry and Molecular Biology 158 117-126. (doi:10.1016/j.jsbmb.2015.12.025)

Rochat B, Morsman JM, Murray GI, Figg WD \& McLeod HL 2001 Human CYP1B1 and anticancer agent metabolism: mechanism for tumorspecific drug inactivation? Journal of Pharmacology and Experimental Therapeutics 296 537-541.

Singh MN, Stringfellow HF, Taylor SE, Ashton KM, Ahmad M, Abdo KR, El-Agnaf OM, Martin-Hirsch PL \& Martin FL 2008 Elevated expression of CYP1A1 and gamma-SYNUCLEIN in human ectopic (ovarian) 
endometriosis compared with eutopic endometrium. Molecular Human Reproduction 14 655-663. (doi:10.1093/molehr/gan056)

Tsai SJ, Wu MH, Lin CC, Sun HS \& Chen HM 2001 Regulation of steroidogenic acute regulatory protein expression and progesterone production in endometriotic stromal cells. Journal of Clinical Endocrinology and Metabolism 86 5765-5773. (doi:10.1210/jcem.86.12.8082)

Tsuchiya Y, Nakajima M \& Yokoi T 2005 Cytochrome P450-mediated metabolism of estrogens and its regulation in human. Cancer Letters $\mathbf{2 2 7}$ 115-124. (doi:10.1016/j.canlet.2004.10.007)

Vandesompele J, De Preter K, Pattyn F, Poppe B, Van Roy N, De Paepe A \& Speleman F 2002 Accurate normalization of real-time quantitative RT-PCR data by geometric averaging of multiple internal control genes. Genome Biology 3 RESEARCH0034. (doi:10.1186/gb-2002-3-7research0034)

Waxman DJ 1988 Interactions of hepatic cytochromes P-450 with steroid hormones. Regioselectivity and stereospecificity of steroid metabolism and hormonal regulation of rat P-450 enzyme expression. Biochemial Pharmacology 37 71-84. (doi:10.1016/0006-2952(88)90756-3)

Zanger UM \& Schwab M 2013 Cytochrome P450 enzymes in drug metabolism: regulation of gene expression, enzyme activities, and impact of genetic variation. Pharmacology \& Therapeutics 138 103-141.

Zhu BT \& Conney AH 1998 Functional role of estrogen metabolism in target cells: review and perspectives. Carcinogenesis 19 1-27. (doi:10.1093/ carcin/19.1.1)

Received 10 December 2015

First decision 28 January 2016

Revised manuscript received 22 February 2016

Accepted 24 March 2016 